\title{
Composición y biomasa fitoplanctónica en zona riparia y lagunar-estuarina del Río San Pedro, Nayarit (2008-2009)
}

\section{Composition and phytoplankton biomass in the riparian and lagoon-estuarine zone of the San Pedro River, Nayarit (2008-2009)}

\author{
ROBLES-JARERO, Elva Guadalupe †*, PÉREZ-PEÑA, Martín y LÓPEZ-URIARTE, Ernesto \\ Universidad de Guadalajara, Laboratorio de Ecosistemas Marinos y Acuicultura, Depto. de Ecología. Centro Universitario \\ de Ciencias Biológicas y Agropecuarias, México. Ramón Padilla Sánchez, 2100, Nextipac, 44600, Zapopan, Jal.
}

ID $1^{\mathrm{er}}$ Autor: Elva Guadalupe, Robles-Jarero / ORC ID: 000-0003-2537-671X, CVU CONACYT ID: 59946

ID $1^{\text {er }}$ Coautor: Martín, Pérez-Peña / ORC ID: 0000-0002-9479-0722, CVU CONACYT ID: 1012062

ID $2^{\text {do }}$ Coautor: Ernesto, López-Uriarte / ORC ID: 0000-0003-0478-6744, CVU CONACYT ID: 62011

DOI: 10.35429/JSI.2019.9.3.18.25

Recibido 16 de Enero, 2019; Aceptado 30 Marzo, 2019

\section{Resumen}

Se estimó la biomasa (Cl-a) y composición del fitoplancton en la parte baja del río San Pedro, Nayarit y zona lagunar-estuarina adyacente (diciembre 2008 - julio 2009). Se muestrearon 9 estaciones para evaluar la biomasa fitoplanctónica ( $\mathrm{Cl}-a)$ en superficie. Las muestras se protegieron de la luz y se filtraron para congelarlas y posteriormente ser analizadas en laboratorio mediante la técnica de Srickland y Parsons (1972). Se determinaron parámetros ambientales (temperatura, salinidad y oxígeno disuelto) con un multímetro YSI 55, la profundidad Secchi, porcentaje de nubosidad y dirección y velocidad del viento. Se realizaron arrastres de fitoplancton con una red de luz malla de $34 \mu \mathrm{m}$, registrándose 19 familias y 39 especies. Las Crysophytas presentaron 16 especies, destacan Fragilaria sp. y Ephitemia zebra, Chlorophytas: 9 familias y 10 especies - Closterium aciculare, Oocystis lacustris y Spirogyra sp., Cyanophytas: 3 familias y 9 especies - Microcystis-aeroginosa, M. flos-aquae, Anabaena flos-aquae y Phormidium tenue y Euglenophytas: 1 familia y 4 especies. Los valores de Cl$a$ resultaron bajos $\left(0.002-0.71 \mathrm{mgCl} a . \mathrm{m}^{-3}\right)$ comparados con sitios cercanos. La mayoría de las estaciones presentaron un alto grado de materia orgánica disuelta que impide la entrada de la luz, indispensable para que el fitoplancton realice la fotosíntesis.

Clorofila- $a$, fitoplancton, Río San Pedro

\begin{abstract}
Biomass $(\mathrm{Cl}-a)$ and phytoplankton composition were estimated in the lower part of the San Pedro, Nayarit and lagoon-estuarine areas (December 2008 - July 2009). Nine stations were sampled to evaluate phytoplankton biomass $(\mathrm{Cl}-a)$ on the surface. The samples were protected from light and filtered to freeze them and subsequently analyzed in the laboratory using the technique of Srickland and Parsons (1972). Environmental parameters (temperature, salinity and dissolved oxygen) were determined with a YSI 55 multimeter, Secchi depth, cloudiness percentage and wind direction and speed. Phytoplankton trawls were carried out with a mesh network of $34 \mu \mathrm{m}$, registering 19 families and 39 species. The Crysophytas presented 16 species, Fragilaria sp. and Ephitemia zebra, Chlorophytas: 9 families and 10 species - Closterium aciculare, Oocystis lacustris and Spirogyra sp., Cyanophytas: 3 families and 9 species - Microcystisaeroginosa, M. flos-aquae, Anabaena flos-aquae and Phormidium tenue and Euglenophytas: 1 family and 4 species. Cl- $a$ values were low (0.002 - $0.71 \mathrm{mgCl} a . \mathrm{m}-3)$ compared to nearby sites. Most of the stations presented a high degree of dissolved organic matter that prevents the entry of light, essential for phytoplankton to perform photosynthesis.
\end{abstract}

Chlorophyll-a, phytoplankton, San Pedro River

Citación: ROBLES-JARERO, Elva Guadalupe, PÉREZ-PEÑA, Martín, LÓPEZ-URIARTE, Ernesto. Composición y biomasa fitoplanctónica en zona riparia y lagunar-estuarina del Río San Pedro, Nayarit (2008-2009). Revista de Innovación Sistemática 2019. 3-9:18-25

\footnotetext{
*Correspondencia al Autor (Correo electrónico: elva.robles@academicos.udg.mx)

$\dagger$ Investigador contribuyendo como primer Autor.
} 


\section{Introducción}

El fitoplancton es la unidad básica de producción de la materia orgánica en los ambientes acuáticos. Al igual que la mayoría de las plantas, las microalgas fijan carbono por medio de la fotosíntesis, a partir del agua, bióxido de carbono y energía luminosa (GonzálezGonzález, 1992). El fitoplancton como productor primario juega un papel importante tanto en la circulación de nutrientes como en el flujo de energía de los ecosistemas acuáticos, porque controla el crecimiento y la capacidad reproductora de las poblaciones de otros organismos acuáticos (Contreras-Espinoza y Warner, 2004).

La productividad también puede estimarse por la clorofila- $a(\mathrm{Cl} a)$, la cual es utilizada universalmente como una medida de la biomasa de fitoplancton. Una vez que se ha generado carbono biogénico en un cuerpo acuático a partir de la actividad fotosintética, éste puede ser transformado por el circuito microbiano dentro de la zona fótica o exportado fuera de la red trófica. En consecuencia, la dinámica del fitoplancton con relación al ambiente local y a otros organismos, es de particular relevancia para estimar la productividad biológica del ecosistema. Como un componente de los procesos biogeoquímicos, la productividad primaria, con valores de $1 \mathrm{~g} \cdot \mathrm{m}^{-}$ ${ }^{2} \cdot \mathrm{d}^{-1}$ en promedio, permite comprender la función del fitoplancton en la bomba de carbono que reduce el $\mathrm{CO}^{2}$ atmosférico.

\section{Justificación}

El presente trabajo formó parte de un estudio diagnóstico para el aprovechamiento de los escurrimientos y el control de avenidas en la cuenca baja del río San Pedro, para la adecuación de la manifestación de impacto ambiental modalidad regional del Proyecto Hidroeléctrico Las Cruces, en el estado de Nayarit. Esto de acuerdo con los lineamientos de la Secretaría de Medio Ambiente y Recursos Naturales Costeros, así como recomendaciones de la Comisión de Sitios RAMSAR, sustentada en listados taxonómicos, abundancia y distribución de organismos acuáticos del plancton y macroinvertebrados bentónicos, evaluación de tasas de productividad acuática y biomasa planctónica y estado actual de la actividad pesquera.
No obstante la importancia del sitio, son escasos los estudios sobre los ambientes acuáticos en este sector, el paisaje acuático corresponde a un paisaje fluvial por esencia, ya que la red de drenaje es muy densa debido al bioclima. Los ambientes lénticos son embalses de origen antropogénico, de los cuales solo se conocen algunos datos ecológicos y listados ícticos. Los resultados sustentarán las condiciones físico-biológicas de esta importante zona y la elaboración de escenarios probables en el mediano y largo plazo, ya que la zona se caracteriza por su alto potencial productivo pero también por su gran fragilidad de acuerdo a la caracterización de las comunidades de manglar existente en la zona costera.

\section{Antecedentes}

El plancton se ha estudiado sistemáticamente desde hace mucho tiempo, pero su investigación se ha incrementado en los años recientes debido al interés por utilizarlo en la acuacultura, así como indicador de cambios ambientales en un tiempo breve, dada su interacción con el agua por su corto ciclo de vida, su rápida respuesta a cambios ambientales, su tamaño pequeño, presentar géneros tolerantes a concentraciones tóxicas de metales pesados y por su gran abundancia, representando una herramienta fundamental en estudios de calidad del agua (SARH, 1983).

Las investigaciones con relación al plancton han sido esencialmente taxonómicas y florísticas realizadas principalmente por extranjeros y sólo recientemente por investigadores mexicanos. Generalmente, en estos estudios se habla de una baja diversidad, donde la comunidad fitoplanctónica está conformada por especies y variedades de cianofitas, clorofitas, euglenofitas y diatomeas (Torres-Orozco Bermeo y Pérez-Rojas, 2002; Oliva-Martínez et al., 2014).

\section{Objetivos}

\section{Objetivo General}

Estimar la biomasa (clorofila- $a$ ) y analizar la composición de las comunidades fitoplanctónicas en la columna iluminada de la zona riparia baja del Río San Pedro y zona lagunar-estuarina adyacente. 


\section{Metodología}

\section{Área de estudio}

El área de estudio forma parte de la cuenca A Río San Pedro, cuya extensión es superior a los $25,000 \mathrm{~km}^{2}$ dentro de la región hidrológica RH11 Presidio - San Pedro. El río San Pedro se considera el séptimo río más caudaloso de México. Nace en la Sierra del Mezquital, en las partes más altas de los estados de Durango y Zacatecas, atraviesa una gran parte del Altiplano mexicano y cruza la sierra a través del cañón del Mezquital y continúa hacia las llanuras costeras de Nayarit, donde se convierte en la arteria principal que suministra agua dulce a Marismas Nacionales, el manglar más extenso del Pacífico mexicano (CONANP, 2013).

En el estado de Nayarit esta cuenca cubre cerca del $16 \%$ de la superficie estatal, con vegetación representativa de selva mediana subcaducifolia en diversos estados de sucesión y con distinto grado de perturbación. En la parte baja predominan las coberturas de origen antropocéntrico, predominantemente para usos agropecuarios y finalmente se presenta una asociación de humedales costeros, destacando un complejo sistema lagunar y marismas con presencia o no de vegetación halófila de tipo emergente, arbustivo y arbóreo. Debido a la baja altitud en esta última sección de la cuenca, el curso del río no está bien definido cerca de la costa, donde se une a un complejo sistema lagunar estuarino.

Este río descarga un promedio de 2735 millones de $\mathrm{m}^{3}$ al año (medido en la estación hidrométrica San Pedro), con un flujo laminar calculado en $106 \mathrm{~mm}$ y un coeficiente de escurrimiento de $7.9 \%$. La temperatura media anual varía de $14^{\circ}$ a $26^{\circ} \mathrm{C}$ y presenta una precipitación anual que varía de 700 a 2000 mm, ubicándose la época de mayor precipitación entre los meses de junio y noviembre. Para el resto del año la precipitación se reduce considerablemente o es nula (CIAD, 2010).

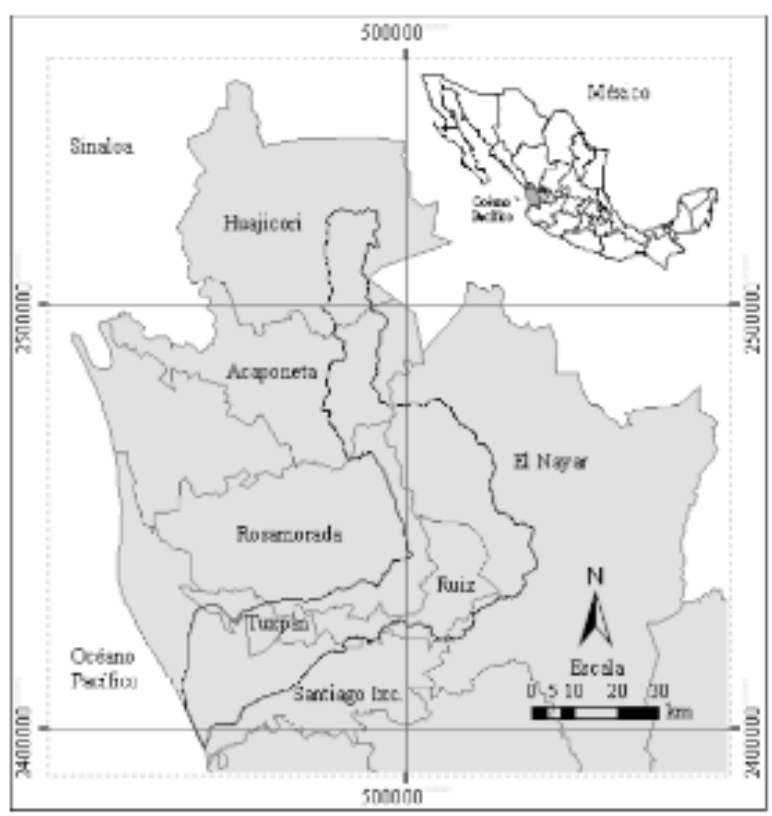

Figura 1 Ubicación de la cuenca hidrológica del Río San Pedro Mezquital

Marismas Nacionales fue decretada como Reserva de la Biósfera en mayo de 2010 por ser un sitio importante para la región por sus recursos pesqueros, el manglar y la gran diversidad biológica que presenta, y fue reconocido como sitio RAMSAR por ser un humedal importante para las aves.

\section{Materiales y métodos}

Los muestreos se realizaron en los meses de diciembre de 2008 a julio de 2009, en estaciones ubicadas en la parte baja del Río San Pedro desde la cortina hacia aguas abajo y en la zona lagunarestuarina. Se seleccionaron estaciones ubicadas en puntos estratégicos señaladas con potencial pesquero. Las coordenadas de estos sitios fueron georeferenciadas con un GPS y aparecen en la tabla 1 .

\begin{tabular}{|l|l|l|}
\hline Localidad & Latitud N & Longitud W \\
\hline Zona riparia & $22^{\circ} 05^{\prime} 38.2^{\prime \prime}$ & $104^{\circ} 56^{\prime} 48.7^{\prime \prime}$ \\
\hline 1 Cortina & $22^{\circ} 02^{\prime} 28.0^{\prime \prime}$ & $104^{\circ} 55^{\prime} 56.1^{\prime \prime}$ \\
\hline 2 Naranjo & $22^{\circ} 02^{\prime} 31.1^{\prime \prime}$ & $104^{\circ} 55^{\prime} 41.0^{\prime \prime}$ \\
\hline 3 San Pedro & $21^{\circ} 53^{\prime} 19.1^{\prime \prime}$ & $105^{\circ} 27^{\prime} 47.7^{\prime \prime}$ \\
\hline \multicolumn{3}{|c|}{ Zona lagunar-estuarina } \\
\hline 4 Mexcaltitan & $21^{\circ} 53^{\prime} 23.2^{\prime \prime}$ & $105^{\circ} 27^{\prime} 39.9^{\prime \prime}$ \\
\hline 5 Puerta del Río & $21^{\circ} 52^{\prime} 26.8^{\prime \prime} 1$ & $105^{\circ} 28^{\prime} 25.5^{\prime \prime}$ \\
\hline 6 Barra de Chalpa & $21^{\circ} 50^{\prime} 28.4^{\prime \prime}$ & $105^{\circ} 28^{\prime} 31.5^{\prime \prime}$ \\
\hline 7Toluca & $21^{\circ} 48^{\prime} 48.2^{\prime \prime}$ & $105^{\circ} 30^{\prime} 09.5^{\prime \prime}$ \\
\hline 8 La Borrega & $21^{\circ} 44^{\prime} 32.2^{\prime \prime}$ & $105^{\circ} 29^{\prime} 35.8^{\prime \prime}$ \\
\hline 9 Boca de Camichín
\end{tabular}

Tabla 1 Coordenadas de las estaciones de muestreo, río San Pedro Mezquital 


\section{Parámetros físico-químicos}

Se tomaron parámetros ambientales y físicoquímicos en la superficie de la columna de agua como temperatura, salinidad y oxígeno disuelto con un multímetro YSI 55, la profundidad del disco de Secchi, el porcentaje de nubosidad y la dirección y velocidad del viento con un anemómetro. Se colectaron muestras de agua de cada estación para determinar clorofila- $a$, las cuales se mantuvieron en refrigeración y posteriormente fueron filtradas mediante un sistema Millipore con filtros de fibra de vidrio $\mathrm{GF} / \mathrm{F}$ de $0.45 \mu \mathrm{m}$ de poro y $25 \mathrm{~mm}$ de diámetro. Los filtros se conservaron congelados hasta su análisis en el laboratorio y se analizaron mediante la técnica espectrofotométrica de Strickland y Parsons (1972). Se efectuaron arrastres de fitoplancton con redes de luz de malla de $34 \mu \mathrm{m}$, para estimar la composición de los principales grupos taxonómicos. Las muestras fueron fijadas inmediatamente con formol al $4 \%$.

\section{Resultados}

Los resultados presentados en la tabla 2, son valores promedio obtenidos de todas las estaciones de muestreo.

\begin{tabular}{|c|c|c|c|c|}
\hline Estación & $\begin{array}{l}\text { Temperatura } \\
{ }^{\circ} \mathrm{C}\end{array}$ & $\begin{array}{l}\text { Oxígeno } \\
\text { mg. } \mathrm{L}^{-1}\end{array}$ & $\begin{array}{l}\text { Profundidad } \\
\text { Secchi (m) }\end{array}$ & 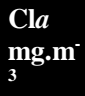 \\
\hline \multicolumn{5}{|c|}{ Zona riparia } \\
\hline 1 & 22.3 & 12.0 & 1.0 & 0.01 \\
\hline 2 & 24.6 & 6.8 & 0.20 & 0.004 \\
\hline 3 & 23.3 & 6.86 & 1.80 & 0.003 \\
\hline \multicolumn{5}{|c|}{ Zona lagunar-estuarina } \\
\hline 4 & 24.9 & 4.2 & 0.30 & 0.056 \\
\hline 5 & 24.2 & 5.0 & 0.10 & 0.005 \\
\hline 6 & 25.8 & 5.6 & 3.8 & 0018 \\
\hline 7 & 26.4 & 8.5 & 0.6 & 0.022 \\
\hline 8 & 26.6 & 11.5 & 0.65 & 0.013 \\
\hline 9 & 25.9 & 10.3 & 1.20 & 0.016 \\
\hline
\end{tabular}

\section{Zona Riparia}

La temperatura del agua varió entre los 22.3 y $32.5^{\circ} \mathrm{C}$, siendo más bajas que la zona estuarina. El oxígeno disuelto fluctuó entre los 12.0 a los $5.8 \mathrm{mg} / \mathrm{L}$, habiendo valores de sobresaturación en la E1 arriba de la cortina, por la velocidad de la corriente. La profundidad secchi registró la misma profundidad de las estaciones 1 y 2 , por ser sumamente someras. La E3, presentó un grado de turbidez leve, pero bajas concentraciones de pigmento, acentuándose esta concentración al nivel de la profundidad del disco de Secchi.
Los valores de clorofila- $a$ registraron variaciones muy marcadas estacionalmente. El mes de enero presentó valores desde no detectados por la técnica (E3), hasta $0.77 \mathrm{mg}$ Cla $\mathrm{m}^{-3}$ en la estación 1. En el mes de marzo los valores fluctuaron de 0.22 a $0.002 \mathrm{mgCl} a . \mathrm{m}^{-3}$ ), siendo particularmente bajos respecto al mes de enero.

\section{Promedio de clorofila- $a$}

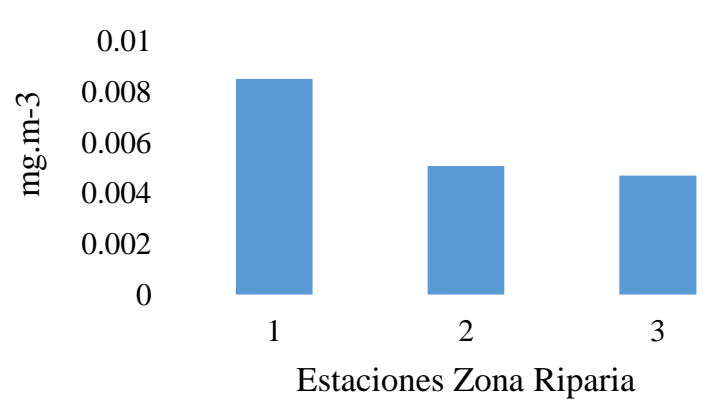

Figura 2 Valores promedios de clorofila-a en estaciones de Río San Pedro

De acuerdo a la dinámica general de los sistemas fluviales, en aguas rápidas predomina la producción primaria sobre la descomposición, siendo el perifiton (aufwuchs) el actor dominante. Se compone principalmente de diatomeas, cianobacterias y musgos adheridos a las piedras o troncos.

Por lo general, en las partes altas de los ríos hay pocos nutrimentos, su productividad primaria es muy baja y su biota depende de las entradas de materia orgánica alóctona, por lo que se consideran heterotróficos o de primer a tercer orden (Vannote et al., 1980). Las condiciones cambian gradualmente aguas abajo al elevarse la concentración de nutrimentos y todo tipo de sustancias, convirtiendo al sistema en autótrofo en las corrientes de cuarto a sexto orden, caso particular la estación 1 de este estudio.

En las corrientes de órdenes superiores, el exceso de sólidos impide la penetración de la luz al sistema y esto, aunado con las altas concentraciones de materia orgánica fina lo vuelve heterótrofo (Vannote et al., 1980). Este cambio en las condiciones del río, puede ocurrir al mezclarse en un mismo cauce aportes de corrientes con diferencias características que al irse uniendo contribuirán a la modificación de las condiciones físicoquímicas, como pudo apreciarse en la estación 2, donde confluyen dos afluentes del río San Pedro. 


\section{Zona estuarina}

Los valores de temperatura superficial fluctuaron entre los 24.2 y $32.9{ }^{\circ} \mathrm{C}$, de acuerdo a la estacionalidad. El oxígeno disuelto presentó un gradiente incrementando gradualmente de norte a sur, debido a la alta demanda de oxígeno de las estaciones cercanas a Isla de Mexcaltitan, asociadas por la gran turbidez del agua en los primeros sitios de muestreo.

Los sistemas lagunares-estuarinos son ecosistemas complejos y dinámicos que intercambian agua y propiedades con los océanos adyacentes por medio de las corrientes de marea. Estos sistemas costeros generalmente son someros y tienen limitadas conexiones con el mar adyacente, resultando fuertes gradientes temporales de propiedades físicas, químicas y biológicas (Nogueira et al. 1997)

Con respecto a la biomasa, en febrero los valores oscilaron desde 0 a $0.71 \mathrm{mg} \mathrm{Cla} \mathrm{m}^{-3}$ registrándose el valor más alto en la estación 1. Para el mes de mayo, la concentración de clorofila- $a$ descendió de manera considerable, registrando un rango entre 0 a $0.29 \mathrm{mg} \mathrm{Cla} \mathrm{m}^{-3}$ (E1). En el mes de julio se registraron también valores menores entre 0.19 a $0.082 \mathrm{mg} \mathrm{Cla} \mathrm{m}^{-3}$ (Fig. 3).

Valores promedio de clorofila-a (marzo-

09)

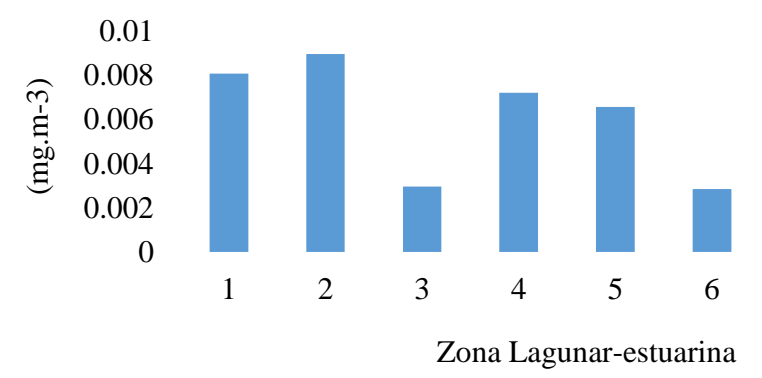

Figura 3 Valores promedios de clorofila-a en estaciones de zona lagunar-estuarina

El grado de turbidez de las estaciones en el mes de julio fluctuó entre los 10 y $25 \mathrm{~cm}$ de profundidad, siendo particularmente bajo debido a la gran resuspensión de sedimentos y materia orgánica por lluvias en esta temporada. Los máximos de productividad durante la temporada de lluvias se relacionan al incremento de nutrientes y materiales húmicos y esta condición es comúnmente registrada en lagunas costeras (Sandoval-Rojo et al., 1988, Flores-Verdugo et al., 1990).
Además, los valores bajos de clorofila-a, se asocian a un acoplamiento importante con el zooplancton. Se ha señalado por varios autores, que las cadenas alimentarias en estas lagunas se caracterizan por dos factores principales: a) una columna de agua rica en fitoplancton (principalmente nanofitoplancton), y b) descomposición de materia orgánica e ingreso de detritus abundante derivado en gran medida de fibras de manglar y otras materias orgánicas, provenientes de las orillas cercanas al delta del río, donde dominaba vegetación de plantas emergentes, sumergidas y herbáceas mixtas (Contreras-Espinoza y Warner, 2004). La gran cantidad de materia orgánica, impide la entrada de luz, indispensable para que el fitoplancton realice el proceso de fotosíntesis, mediante el cual se elaboran alimentos para el resto de los organismos y se proporciona oxígeno para la respiración de los organismos que habitan en el agua (Barreiro Güemes y Signoret-Poillon, 1999).

Las partículas suspendidas en la columna pueden darles un color particular, cuando la coloración no es intensa, el sistema posee una capa de agua superior con capacidad de producir alimento y oxígeno, si el agua es turbia, por la acción de sólidos suspendidos, disminuye su capacidad productiva en la medida en que disminuye la profundidad de la luz.

Uno de los sitios cercanos a este sistema lagunar es Teacapán-Agua Brava, considerado un ecosistema importante en el estado de Nayarit, con una tasa neta anual promedio de productividad primaria acuática, calculada en $0.41 \mathrm{~g} \mathrm{C}^{-\mathrm{m}^{-3}} \cdot \mathrm{d}^{-1}$. La tasa neta de productividad primaria acuática presenta amplias variaciones mensuales, con máximos en febrero, marzo, junio y agosto. El primer máximo de productividad en febrero y marzo coincide con: la influencia nerítica que se da en esta época a consecuencia de la predominancia de vientos del noroeste; florecimientos de fitoplancton costero registrados en la costa del Pacífico (SandovalRojo et al., 1988), y mayor incidencia debido a la máxima irradiancia anual de luz y mayor transparencia del agua (Flores-Verdugo et al., 1990). El valor de la productividad de TeacapánAgua Brava resulta menor al registrado en lagunas-estuarinas del Pacífico mexicano, como es el caso del Estero de Urías, que presenta condiciones de eutrofización antopogénica (Robles-Jarero, 1985). 


\section{Composición de la comunidad planctónica}

El análisis de las muestras obtenidas en los meses de diciembre 2008 y febrero 2009, señala la presencia de 19 familias y 39 especies (Figura 4). El grupo de las Crysophytas fue el más numeroso (16 especies), donde destacan la presencia de Fragilaria sp. y Ephitemia zebra. Las Chlorophytas contribuyeron con 10 especies y 9 familias.

\section{Riqueza de especies de fitoplancton}

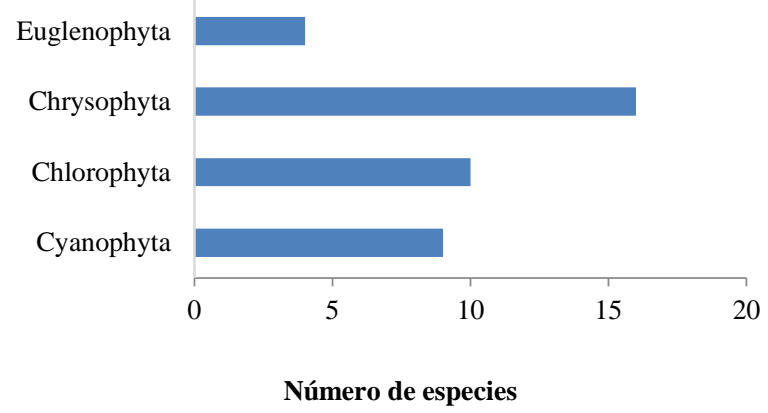

Figura 4 Número de especies en los principales grupos taxonómicos de fitoplancton

Las especies Closterium aciculare, Oocystis lacustris y Spirogyra sp fueron las más importantes del grupo. Las Cyanophytas presentaron 9 especies en 3 familias, predominaron las especies Microcystisaeroginosa, M. flos-aquae, Anabaena flosaquae y Phormidium tenue, en casi todas las estaciones de muestreo. Las Euglenophytas fueron menos abundantes en esta época, con 4 especies y una familia (Figura 5).

\section{Familias del fitoplancton}

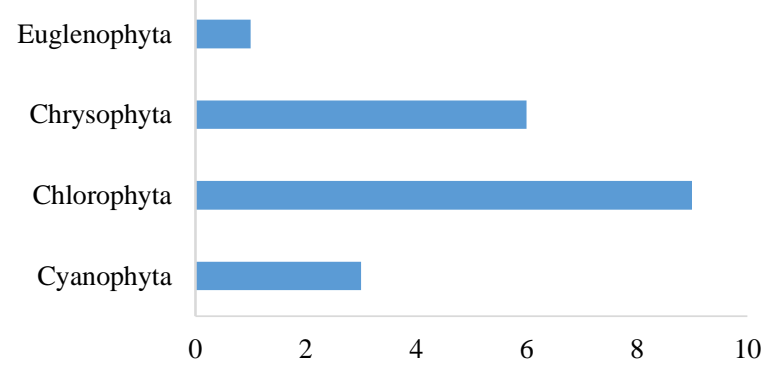

Figura 5 Total de familias de fitoplancton

La contribución de diatomeas y clorofitas fue más importante que el resto de los grupos (Figura 6).

\section{Porcentaje de especies de fitoplancton}

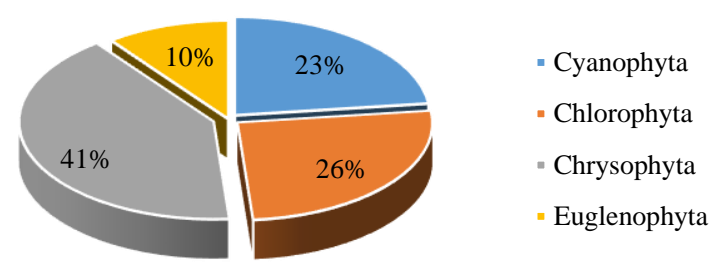

Figura 6 Contribución de los principales grupos taxonómicos en la comunidad fitoplanctónica

La presencia de especies como Anabaena flos-aquae, Microcystis aeroginosa, Microcystis flos-aquae, indican condiciones de betamesosaprobiedad o de contaminación moderada (De la Lanza et al, 2000) en la zona cercana a la cortina. sin embargo, dominaron las diatomeas y clorofitas en gran medida en todas las estaciones, siendo grupos característicos de aguas limpias, frías o cálidas y de gran importancia en la alimentación de peces y crustáceos.

En el interior de las lagunas costeras, los procesos biogeoquímicos son extremadamente complejos debido a las interacciones con los sedimentos del fondo (Fourqurean et al. 1993), los cuales son fuentes de nitrógeno y fósforo inorgánico a la columna de agua. La sedimentación en las lagunas costeras es uno de los principales factores que regulan su producción (Lohse et al. 1993), a menudo en asociación con la pérdida de fósforo orgánico particulado en la columna de agua.

Las variaciones estacionales del fitoplancton indican generalmente valores mínimos de productividad primaria en invierno y máximos en primavera, controladas fundamentalmente por la dimensión de la zona iluminada. Esta depende de la disponibilidad de luz durante el año y de factores hidrológicos e hidrográficos como variaciones de caudal de los afluentes y del comportamiento térmico (Montecino y Cabrera, 1984; Cabrera y Montecino, 1982). Al comparar los resultados obtenidos en los embalses se observan gradientes respecto a la dimensión de la zona eufótica, biomasa fitoplanctónica (expresada como Clorofila- $a$ ) y actividad fotosintética. 


\section{Agradecimiento}

Este estudio formó parte del Proyecto Hidroeléctrico Las Cruces, para la caracterización ecológica de la parte baja del Río San Pedro hasta su desembocadura en el Océano Pacífico, más la porción norte de la zona reconocida como sitio RAMSAR, por acuerdo entre la Comisión Nacional del Agua (CNA) y la Comisión Federal de Electricidad (CFE).

\section{Conclusiones}

Los valores de Clorofila- $a$ resultaron bajos con respecto a otros sitios cercanos, principalmente por el acoplamiento con el zooplancton, así como por la gran cantidad de detritus suspendido en la columna de agua.

Los grupos más representativos de la composición fitoplanctónica fueron diatomeas y clorofitas, que son característicos de cuerpos de agua con características betamesotróficas o de moderada contaminación.

Las observaciones del proyecto global sobre los efectos de la construcción de la presa Las Cruces en el Río San Pedro, señalaban la retención de grandes cantidades de sólidos suspendidos y de carbono orgánico, ambos elementos indispensables para mantener el equilibrio en los ecosistemas costeros, tanto de marismas como manglares.

En estudios recientes representantes de organizaciones civiles señalaron que la Secretaría de Energía ha retirado el proyecto hidroeléctrico Las Cruces del programa para el Desarrollo del Sistema Eléctrico Nacional (Prodesen 2016-2030); y propone en su lugar una diversificación de la matriz eléctrica con tecnología renovable. La apertura del sector eléctrico a la inversión privada y las tendencias internacionales de generación de energía con fuentes renovables, han cambiado las proyecciones de la Sener respecto al proyecto hidroeléctrico Las Cruces.

\section{Referencias}

Barreiro-Güemes, M. A. y Signoret-Poillon M. (1999). Productividad Primaria en los Sistemas Acuáticos Costeros, Métodos de Evaluación. Universidad Autónoma Metropolitana. México.
Banderas-Tarabay, A. y González-Villela, R. (2002). La investigación limnológica y el manejo de los recursos acuáticos. En: De la Lanza-Espino G. y García-Calderón, J. L. (compiladores). Lagos y presas de México, AGT editor S. A. 2002. p. 621-645.

Cabrera, S. y Montecino V. (1982). Eutrophy in Lake Aculeo, Chile. Plant and Soil; 67: 377-387.

CONANP (2013). Comisión Nacional de Áreas Naturales Protegidas. Gobierno de México.

Contreras-Espinosa, F. y Warner, B. G. (2004). Ecosystem characteristics and management considerations for coastal wetlands in Mexico. Hydrobiologia 511: 233-245.

CIAD (2010). Inventario de Humedales de la subcuenca San Pedro y alternativas para su manejo y conservación en función de las tendencias de cambio de usos de suelo y patrones de escurrimiento-Informe Técnico Final.

De la Lanza, E.G. y Hernández, P.S y Carbajal Pérez J. L. (2000). Organismos indicadores de la calidad del agua y de la contaminación (bioindicadores). Plaza y Valdes Ed., México, D.F.

Flores-Verdugo, F., González-Farías, F., Ramírez-Flores, O., Amezcua-Linares, F., A. Yañez-Arancibia, A., Alvarez-Rubio,M. y Day, J. W. (1990). Mangrove Ecology, Aquatic Primary Productivity and Fish Community Dynamics in the Teacapán-Agua Brava LagoonEstuarine System (Mexican Pacific). Estuaries 13:219-230.

Fourqurean, J. W., Jones, R. D y Zieman, J. C. (1993). Processes influencing water column nutrient characteristics and phosphorus limitation of phytoplankton biomass in Florida Bay, FL, USA: Interferences from spatial distributions. Estuarine, Coastal and Shelf Science

36: 295-314.

González-González, J. (1992). Flora ficológica de México: Cconcepciones y estrategias para la integración de una flora ficológica nacional. Ciencias; 6: 13-33. 
Montecino, V. y Cabrera S. (1984). Limnological pilot project for the characterization of temperate lakes in Central Chile. p. 22 pp.

Nogueira, E, Perez, F. F y Ríos, A. F. (1997). Seasonal patterns and long-term trends in an estuarine upwelling ecosystem (Ría de Vigo, NW Spain). Estuarine, Coastal and Shelf Science 44: 285-300.

Oliva-Martínez, M. G., Godínez-Ortega, J. L y Zuñiga-Ramos, C. A (2014). Biodiversidad del fitoplancton de aguas continentales en México. Revista Mexicana de Biodiversidad, Supl. 85:S54-S61.

Robles-Jarero, E. G. (1985). Productividad primaria del fitoplancton y distribución temporo-espacial de pigmentos fotosintéticos (clorofila "a" y feopigmentos) en el Estero de Urías, durante un ciclo anual (septiembre 1982agosto 1983). Tesis profesional, Escuela de Biología, Universidad Autónoma de: 76 p.

Sandoval-Rojo. L.F., F. Flores-Verdugo, U. Saragoza-Araujo, J.W. Day, y A. EstradaMercado. (1988). Phytoplankton Productivity in the Barra de Navidad Coastal Lagoon on the Pacific Coast of Mexico. Revue D'Hydrobiologie Tropicale 21:101-108.

SARH (1983). Secretaría de Agricultura y Recursos Hdraúlicos. Gobierno de México.

Strickland, J. H. D y Parsons, T, R. (1972). A Practical Handbook of Seawater Analysis. Fisheries Research Board of Canada, 315 pp.

Torres Orozco Bermeo R. E. y Pérez Rojas A. El lago de Catemaco. (2002). En: De la Lanza Espino, G y J. L. García Calderón (compiladores). AGT editor S. A. México. p. 213-251.

Vannote, R. L., Minshall, G. W., Cummins, K. W., Sedell, J. R y Cushing, C. E (1980). The river continuum concept. Can. J. Fish. Aquat. Sci. 37: 130-137. 